\title{
BIOPROSPECCIÓN ANTÁRTICA: ¿HACIA UNA INSTITUCIONALIDAD JURÍDICA SUI GÉNERIS?*
}

\section{ANTARCTIC BIOPROSPECTION: MARCHING TOWARDS A SUI GENERIS LEGAL INSTITUTIONALITY?}

Fernando Villamizar-Lamus**

Fecha de recepción: 23 de julio de 2014 Fecha de aceptación: 15 de diciembre de 2014 Disponible en línea: 30 de junio de 2015

\section{Para citar este artículo/To cite this article}

\author{
Villamizar-Lamus, Fernando, Bioprospección antártica: ¿hacia una ins- \\ titucionalidad jurídica sui géneris?, 130 Vniversitas, 309-346 (2015). http:// \\ dx.doi.org/10.11144/Javeriana.vj130.bahi \\ doi:10.11144/Javeriana.vj130.bahi
}

* Artículo de revisión

** Abogado, Universidad del Rosario. Magíster en derecho de la empresa, Pontificia Universidad Católica de Chile. Doctor en ciencia política y sociología, Universidad Pontificia de Salamanca. Investigador del Observatorio Regional de Paz y Seguridad, Universidad Bernardo O'Higgins. Profesor, Facultad de Derecho, Universidad de los Andes (Chile). Contacto: fvillamizar@ miuandes.cl, fvillamizar@ubo.cl 


\section{RESUMEN}

La bioprospección en la Antártida es una actividad que día a día toma mayor importancia, por sus implicaciones geopolíticas y porque, de ser exitosa, puede generar sumas millonarias en utilidades para quienes la realicen. En ese contexto, el objetivo del presente artículo es hacer una revisión de cómo se ha abordado la regulación jurídica de la bioprospección antártica y de cómo se ha empezado a generar de manera incipiente cierta institucionalidad para su regulación. La metodología empleada se basa en una revisión documental de las principales propuestas académicas y documentos del Sistema del Tratado Antártico, STA, y de las bases de datos que registran las actividades de bioprospección antártica. De la revisión realizada, se puede concluir que ciertas propuestas para regular la bioprospección antártica han sido acogidas en alguna medida e implementadas por entes relacionados con el STA pero no propios de este, lo cual genera una institucionalidad reguladora sui géneris. Esto implica entonces que el STA como tal está en mora de abordar integralmente toda la problemática alrededor de este tema.

Palabras clave: Sistema del Tratado Antártico; bioprospección antártica; discusión teórica de la regulación de la bioprospección antártica; incipiente institucionalidad reguladora de la bioprospección antártica 


\section{ABSTRACT}

Antarctic bioprospection is an activity that becomes more and more important with each passing day due to its geopolitical implications. Also, because should it be successful, it could generate massive profits for those performing it. In this context, the purpose of this article is to make a review of how the legal regulation of the Antarctic bioprospection has been performed and also about the beginning of a certain emerging institutionality for its regulation. The methodology used is based on a document review of the main academic proposals and the documents of the Antarctic Treaty System (ATS), as well as of the databases that record the antarctic bioprospection activities. From the review performed we conclude that certain proposals to regulate Antarctic bioprospection have been accepted in some measure and have been implemented by entities related to the ATS, but not fully part of it, which creates a sui generis institutionality. This implies that the ATS as such still has to fully address the issues related to this topic.

Keywords: Antarctic Treaty System; Antarctic bioprospection; theoretical discussion on the regulation of Antarctic bioprospection; emerging institutionality for the regulation of Antarctic bioprospection

\section{SUMARIO}

Introducción.- I. El Sistema del Tratado Antártico y la bioprospección antártica.- A. Del Sistema del Tratado Antártico (STA).- B. La bioprospección en el Sistema del Tratado Antártico.- II. LA DISCUSIÓN SOBRE LA REGULACIÓN DE LA BIOPROSPECCIÓN ANTÁRTICA.- $A$. ¿Qué se ha discutido en el STA sobre la bioprospección?- B. Las etapas en el análisis jurídico de la bioprospección antártica.- C. La primera etapa del análisis jurídico de la bioprospección antártica.- D. La segunda etapa del análisis jurídico de la bioprospección antártica.- III. INCIPIENTE INSTITUCIONALIDAD DE LA bioprospección antártica del Sistema del Tratado Antártico.- $A$. Proyecto Micromat.- B. Bases de datos sobre bioprospección antártica.- $C$. Herramientas que sirven de fuente para las bases de datos de bioprospección antártica.- D. Depósito o banco de muestras biológicas (ex situ collections).E. Presentaciones de actividades de los estados que realizan bioprospección antártica.- ConClusiones.- Bibliografía. 


\section{INTRODUCCIÓN}

Dadas las particulares condiciones ambientales de la Antártida, el interés científico por esta es creciente y abarca diferentes ámbitos, entre ellos el biológico ${ }^{1}$, cuya investigación se encuadra en la noción de bioprospección, entendiendo por tal, en ausencia de un concepto propio del Sistema del Tratado Antártico (STA), la búsqueda de los productos del metabolismo o de los genomas de los seres vivos para su utilización en procesos industriales o biotecnológicos que redunden en un beneficio para quienes los usufructúen ${ }^{2}$.

Como es de suponer, la realización de actividades bioprospectivas en zonas tan difíciles implica un acervo financiero, tecnológico y logístico que solo algunos Estados pueden desplegar ${ }^{3}$. Para la explotación de recursos en zonas de difícil acceso y no sometidas a jurisdicción nacional diferentes al territorio antártico ${ }^{4}$, algunos países desarrollados sostienen que el Estado descubridor de los recursos genéticos retiene la propiedad de los resultados de la investigación ${ }^{5}$. A esto se han opuesto varios Estados en vías de desarrollo, porque consideran que todos los recursos genéticos en zonas fuera de la jurisdicción nacional y la información que de estos se pueda obtener son patrimonio de la humanidad ${ }^{6}$.

1 Para profundizar sobre la dificultad de la investigación en materia biológica en la Antártida, puede consultar, entre otros, Reunión Consultiva del Tratado Antártico XXVIII (Stockholm, 2005), La bioprospección en la Antártida, Information Paper 8, Agenda Item ATCM18. Disponible en: http://www.ats.aq/devAS/ats_meetings_documents.aspx?lang=e

2 Reunión Consultiva del Tratado Antártico XXIX (Edinburgh, 2006), Progress During 2005-2006 in Implementing the International Polar Year 2007-2008, Information Paper 12, Agenda Item ATCM 18, 1. Disponible en: http://www.ats.aq/devAS/ats_meetings_documents. aspx?lang=s

3 Para profundizar este tema, MeLISSA WeBer, Accreditation as a Regulatory Option for Antarctic Bioprospecting, 42 Polar Record, 4, 349-357 (2006).

4 Sobre la tendencia general en esta materia, BRENDAN COOLSAET, Vers un partage équitable des avantages de la richesse génétique mondiale? Pouvoir et influence des pays les moins avancés dans la gouvernance internationale de la biodiversité (Dynamiques Internationales, Décembre, Working paper \#4, 2011). Disponible en: http://biogov.uclouvain.be/staff/coolsaet/2011\%20 Coolsaet $\% 20-\% 20$ Vers $\% 20$ un $\% 20$ partage $\% 20$ equitable.pdf

5 Para profundizar esta tendencia, consultar, entre otros, Richard J. McLaughlin, Foreign Access to Shared Marine Genetic Materials: Management Options for a Quasi-Fugacious Resource, 34 Ocean Development \& International Law, 3-4, 297-348 (2003).

6 Kirsten E. Zewers, Bright Future for Marine Genetic Resources, Bleak Future for Settlement of Ownership Rights: Reflections on the United Nations Law of the Sea Consultative Process on Marine Genetic Resources, 5 Loyola University Chicago International Law Review, 2, 151-176, 170-172 (2008). Disponible en: http://lawecommons.luc.edu/cgi/viewcontent. cgi? article $=1066 \&$ context $=$ lucilr 
Gracias a las regulaciones contenidas en el STA en la Antártida, esta discusión sobre la propiedad de los recursos genéticos no ha tenido la vehemencia que en otras zonas del planeta no sometidas a una jurisdicción nacional. Sin embargo, la discusión sobre la bioprospección antártica se ha venido planteando en el STA desde la vigésimo quinta reunión del Comité Científico para la Investigación Antártica (Scientific Committee on Antarctic Research, SCAR), celebrada en Concepción (Chile) en julio de 1998. Hasta ahora, el STA ha promulgado varias resoluciones ${ }^{7}$ para abordar su problemática y darle un marco jurídico-institucional.

Por ejemplo, la Resolución 6 (2013) compromete a los Estados parte a afirmar que el Sistema Antártico es el marco jurídico apropiado para regular las actividades de prospección biológica (de que trata la Resolución 9 (2009) como "recolección y uso de material biológico"), y lo hace en los siguientes términos literales: "Los representantes (...) reafirman que el Sistema del Tratado Antártico es el marco apropiado para manejar la recolección de material biológico en el Área del Tratado Antártico y para considerar su uso".

Visto esto, surge la pregunta base del problema de investigación de este artículo: si el STA es el llamado a regular la bioprospección antártica, como lo plantean las Resoluciones 7 (2005), 9 (2009) y 6 (2013), ¿cuál es la institucionalidad que en la práctica está asumiendo esa función? La hipótesis para abordar este problema consiste en sostener que se está generando paulatinamente una institucionalidad reguladora de la bioprospección antártica, que todavía es incipiente, débil, poco integrada y sin la obligatoriedad jurídica requerida, dados todos los intereses que hay en juego.

Para comprobar la hipótesis planteada, primero se revisarán el origen y el desarrollo del STA, y la forma en que se relaciona la bioprospección antártica en ese ámbito. El objetivo de este recuento es brindar un contexto general sobre los conceptos e instituciones involucrados en la problemática planteada. Posteriormente, se

7 La Resolución 6 (2013) recuerda lo establecido en las Resoluciones 7 (2005) y 9 (2009). Sistema del Tratado Antártico, Resolución 6, Prospección biológica en la Antártida (2013). Disponible en: http://ats.aq/documents/ATCM36/fr/ATCM36_fr001_s.pdf. Sistema del Tratado Antártico, Resolución 7, La prospección biológica en la Antártida (2005). Disponible en: http://www.ats. aq/documents/other/atcm28_res7_s.pdf. Sistema del Tratado Antártico, Resolución 9, Recolección y uso de material biológico antártico (2009). Disponible en: http://www.ats.aq/devAS/ info_measures_listitem.aspx?lang=s\&id=450 
abordarán las propuestas encaminadas a regular el fenómeno, con el objetivo de ilustrar el estado del arte sobre las propuestas realizadas para realizar esta regulación. En tercer lugar, se estudiarán los distintos mecanismos que han empezado a regular la bioprospección antártica, cuyo objetivo es comprobar la hipótesis planteada; y finalmente, se expondrán las conclusiones.

\section{EL SISTEMA DEL TRATADO ANTÁRTICO Y LA BIOPROSPECCIÓN ANTÁRTICA}

\section{A. Del Sistema del Tratado Antártico (STA)}

El hito fundacional del STA es precisamente, como su nombre lo sugiere, el Tratado Antártico (TA) ${ }^{8}$, que fue consecuencia de la Conferencia de Washington celebrada entre octubre y diciembre de 1959, y puso fin a una serie de disputas encaminadas a fijar qué Estado se apropiaba de territorios y recursos del continente blan$\mathrm{co}^{9}$. En su estructura se establece una serie de principios dictados con el fin de dirigir las relaciones antárticas, y unos mecanismos destinados a posibilitar la ejecución de estos ${ }^{10}$.

Los principios fundamentales del TA son: (i) el uso pacífico del territorio antártico, (artículo I); (ii) la libertad para la realización de investigación científica en la Antártica de conformidad a lo aplicado en el Año Geofísico Internacional [1 de julio de 1957-31 de diciembre de 1958], evento primordial para posibilitar la negociación del TA (artículo II); (iii) la cooperación internacional en la investigación científica en la Antártida (artículo III); (iv) mientras el Tratado Antártico esté vigente, las reclamaciones territoriales quedan suspendidas, ningún Estado puede realizar nuevas reclama-

8 Tratado Antártico, suscrito en Washington, el 1 de diciembre de 1959. Disponible en: http:// www.ats.aq/documents/ats/treaty_original.pdf. El Tratado Antártico entró en vigor en 1961.

9 Sobre la evolución de las relaciones antárticas, Fernando Villamizar-Lamus, Del descubrimiento a la diplomacia: las etapas históricas de las relaciones antárticas, 10 Tradición y Saber, 2, 51-64 (2013). Disponible en: http://www.academia.edu/8595672/Del_descubrimiento_a_la_diplomacia_las_etapas_de_las_relaciones_ant $\%$ C $3 \%$ Alrticas

10 Para profundizar sobre los principios y mecanismos de protección antártica, FERNANDO Villamizar-Lamus, Tratado Antártico y mecanismos de protección del territorio antártico, 21 International Law, Revista Colombiana de Derecho Internacional, 255-295 (2012). Disponible en: http://www.redalyc.org/pdf/824/82425523009.pdf 
ciones territoriales y ningún acto realizado en la Antártida puede imputarse como ejercicio de soberanía (artículo IV) ${ }^{11}$.

Dado que en sus inicios el TA era un acuerdo de principios básicos con una visión geoestratégica, con el devenir de las relaciones antárticas se hicieron notorios varios vacíos en materia medioambiental, de manejo de la biodiversidad y explotación de minerales. En consecuencia, al TA se han añadido varios instrumentos jurídicos que constituyen el Sistema del Tratado Antártico (STA), cuyo objetivo es generar las bases de gobernanza para administrar la Antártida $^{12}$. Entre los instrumentos más destacados que componen el STA están las Medidas Convenidas para la Protección de Flora y Fauna Antártica (Bélgica, 1964), la Convención para la Conservación de las Focas Antárticas (Londres, 1972), la Convención para la Conservación de los Recursos Vivos Marinos Antárticos (Camberra, 1980) y el Protocolo al Tratado Antártico sobre la Protección del Medio Ambiente (Madrid, 1991), mejor conocido como Protocolo de Madrid ${ }^{13}$.

Para dar cumplimiento a lo establecido en el TA y los instrumentos mencionados, en las reuniones consultivas, el órgano más importante del STA, los miembros consultivos ${ }^{14}$ administran la

11 Hay otro principio, el de consenso para la toma de decisiones, pero a diferencia de los principios mencionados carece de un sustento normativo expreso, aunque hay autores que encuentran aplicación de este principio en varios artículos del Tratado Antártico, como el IX o XII. STUART Kaye, Michael Johnson \& Rachel Baird, Law, en Australia and the Antarctic Treaty System. 50 Years of Influence, 97-117, 101-102 (MARCus Haward \& ToM GrifFiths, eds., University of New South Wales Press Ltd., Sidney, 2011).

12 Para profundizar sobre el desarrollo e instrumentos que hacen parte del Sistema del Tratado Antártico, Fernando Villamizar-Lamus, La bioprospección antártica: indefiniciones e incompatibilidades con el Sistema del Tratado Antártico, 23 International Law, Revista Colombiana de Derecho Internacional, 53-86 (2013). Disponible en: http://www.scielo.org.co/pdf/ilrdi/n23/ n23a03.pdf

13 Medidas Convenidas para la Protección de Flora y Fauna Antártica, Bélgica, 1964. Disponible en: http://www.ats.aq/documents/recatt\%5Catt080_s.pdf. Convención para la Conservación de las Focas Antárticas, Londres, 1972. Disponible en: http://www.ats.aq/documents/recatt/ att076_s.pdf. Convención para la Conservación de los Recursos Vivos Marinos Antárticos, Camberra, 1980. Disponible en: http://www.ats.aq/documents/ats/ccamlr_s.pdf. Protocolo al Tratado Antártico sobre la Protección del Medio Ambiente - Protocolo de Madrid, Madrid, 1991. Disponible en: http://www.ats.aq/documents/recatt/att006_s.pdf

14 De conformidad con el artículo IX del Tratado Antártico (TA), los miembros del TA pueden ser consultivos y no consultivos. Las partes que tienen el carácter de consultivas son aquellos fundadores del STA (Argentina, Australia, Bélgica, Chile, Francia, Japón, Nueva Zelanda, Noruega, Sudáfrica, Unión de Repúblicas Socialistas Soviéticas — hoy Rusia ha tomado su rol-, el Reino Unido de Gran Bretaña e Irlanda del Norte y Estados Unidos) y aquellos Estados que demuestren "su interés en la Antártida mediante la realización en ella de investigaciones científicas importantes, como el establecimiento de una estación científica o el envío de una expedición científica". Actualmente, hay 29 miembros consultivos y 23 no consultivos. 
Antártida, mediante decisiones, resoluciones y medidas, que en conjunto con el trabajo de instituciones como el Comité de Protección Ambiental (Committee for Environmental Protection, CEP), el Comité Científico para la Investigación Antártica (Scientific Committee on Antarctic Research, SCAR) y los observadores, permiten la gobernanza antártica.

\section{B. La bioprospección en el Sistema del Tratado Antártico}

Ahora bien, en este marco jurídico-institucional que brinda el STA y en virtud del principio de libertad de investigación, hay una gran ebullición en materia de investigación científica en la Antártida, en especial de las investigaciones científicas con fines comerciales, y entre ellas con un papel muy destacado, la bioprospección. Para tener una referencia cuantitativa de este auge de la bioprospección antártica, basta citar las cifras de un estudio de la Universidad de las Naciones Unidas, según el cual entre 1988 y 1989 había una sola patente de invención procedente de la bioprospección antártica ${ }^{15}$, mientras que para octubre de 2013 la página web www. bioprospector.org registra 185 patentes, y el Information Paper 22 de la XXXVI Reunión Consultiva del Tratado Antártico (RCTA) (Bruselas, 2013 ${ }^{16}$ ) da cuenta de al menos 439 especies antárticas en patentes. Todo esto en un contexto en el cual la bioprospección en general tiene un gran auge. Solo Estados Unidos, Europa (incluidos Suiza y Noruega, que no son parte de la Unión Europea) y Canadá, entre todos, invirtieron más de 25.000 millones de dólares en $2012^{17}$.

A lo anterior se debe añadir que el ámbito de aplicación de los resultados de la bioprospección es inmensurable. Solo para ejemplificar este punto, vale la pena consignar algunos de los casos. En el

15 Dagmar Lohan \& Sam Johnston, Bioprospecting in Antarctica, United Nations University Institute of Advanced Studies, Yokohama (2005). Disponible en: http://i.unu.edu/media/unu. edu/publication/28551/antarctic_bioprospecting-3.pdf

16 Reunión Consultiva del Tratado Antártico XXXVI (Brussels, 2013), An Update on Status and Trends Biological Prospecting in Antarctica and Recent Policy Developments at the International Level. Information Paper 22, Agenda Item 17. Disponible en: http://www.ats.aq/devAS/ ats_meetings_documents.aspx?lang=s

17 Ernst \& Young, Beyond Borders: Global Biotechnology Report 2012. Disponible en: www. ey.com/Publication/vwLUAssets/Beyond_borders_2012/\$FILE/Beyond_borders_2012.pdf, http://www.ey.com/GL/en/Industries/Life-Sciences/Beyond-borders---global-biotechnologyreport-2012 
campo médico, la explotación comercial del ADN de la enzima del Thermus Aquaticus ha generado una industria en Estados Unidos que asciende a 300 millones de dólares. En el ámbito industrial, el mercado global de enzimas es de unos 50.000 millones de dólares al año, y tiene un crecimiento anual de entre 3 y 5 por ciento. En la industria alimenticia, las glicoproteínas de los peces antárticos son muy apetecidas por sus propiedades anticongelantes y las lipasas tienen una gran demanda para la fermentación de productos como quesos y cervezas. En la industria de cosméticos, la pseudoterosina de diferentes agentes biológicos antárticos es requerida para cremas antiarrugas y ciertas glicoproteínas para tratamientos capilares ${ }^{18}$.

Como se puede apreciar de estos ejemplos, lo que está en juego con la bioprospección antártica, tanto en el ámbito económicofinanciero, como en el político y científico, tiene grandes repercusiones en diversas industrias, en el mejoramiento de la calidad de vida de la humanidad y en las reclamaciones territoriales realizadas en la Antártida ${ }^{19}$. En ese orden de ideas, la lógica indicaría que se debería construir una institucionalidad fuerte para regular estos temas tan sensibles para la humanidad, pues de esa manera todos los interesados tendrían un campo relativamente cierto de acción. Sin embargo, como se verá, el tema es tan complejo y los avances muy pocos, de manera que el camino por recorrer es aún muy largo y no exento de dudas, temores y cuestionamientos.

18 Pamela L. Schoenberg, A Polarizing Dilemma: Assessing Potential Regulatory Gap-Filling Measures for Arctic and Antarctic Marine Genetic Resource Access and Benefit Sharing, 42 International Law Journal, 2, 271-299, $277-279$ (2009). Disponible en: http://scholarship.law. cornell.edu/cgi/viewcontent.cgi?article $=1748 \&$ context $=$ cilj

19 Este aspecto es relevante, porque según el Convenio sobre la Diversidad Biológica de 1992, cuando se hace bioprospección dentro de un Estado se debe contar con el consenso de ese Estado y se deben repartir los beneficios. Dado el régimen particular de la Antártida, las reclamaciones territoriales están suspendidas, pero los Estados reclamantes muy probablemente no pretenden dejar a la deriva los recursos de las zonas reclamadas y los potenciales beneficios que de estos se puedan obtener. Convenio sobre la Diversidad Biológica, Nairobi, 1992. Disponible en: https://www.cbd.int/convention/text/, https://www.cbd.int/doc/legal/ cbd-es.pdf 


\section{LA DISCUSIÓN SOBRE LA REGULACIÓN DE LA BIOPROSPECCIÓN ANTÁRTICA}

\section{A. ¿Qué se ha discutido en el STA sobre la bioprospección?}

La discusión sobre la bioprospección en el STA ha girado en torno a (i) buscar una definición o concepto universalmente aceptado de las actividades que se deben considerar como tal, para posteriormente buscar alguna forma de regulación; (ii) a resaltar los intereses y contradicciones en juego; $y$, (iii) a anunciar mecanismos que se están implementando por entidades relacionadas con el STA, pero no partes integrales de él, para regularla. El sustento de la discusión en el STA han sido propuestas académicas, acogidas por algún Estado para ser presentadas en las Reuniones Consultivas del Tratado Antártico (RCTA).

Esto quiere decir que la discusión académica y la discusión política en el STA son mayoritariamente coincidentes, pues esta se sustente en aquella. Por esta razón y para no reiterar, a continuación se estudiarán las propuestas académicas que han sido llevadas al STA. Las propuestas de los Estados que no han sido formuladas previamente en el ámbito académico se mencionarán expresamente.

\section{B. Las etapas en el análisis jurídico de la bioprospección antártica}

Ante la carencia de un concepto de bioprospección en el ámbito del STA, hay estudios por los cuales se puede presumir que esta ha tenido lugar desde los años sesenta del siglo $\mathrm{XX}^{20}$. No obstante

20 Entre los trabajos científicos publicados más relevantes están los de PAUL R. Burkholder \& EnriQue F. Mandelli, Carbon Assimilation of Marine Phytoplankton in Antarctica, 54 Botany, 437-444 (1965). Disponible en: http://www.ncbi.nlm.nih.gov/pmc/articles/PMC219685/pdf/ pnas00160-0103.pdf. AleXander Scott CAMERon, Staphylococcal Epidemiology in Antarctica, 68 The Journal of Hygiene, 1, 43-52 (1970). Disponible en: http://www.ncbi.nlm.nih.gov/pmc/ articles/PMC2130780/pdf/jhyg00091-0049.pdf. Roy E. CAMERon \& RoBert E. BenoIt, Microbial and Ecological Investigations of Recent Cinder Cones, Deception Island, Antarctica. A Preliminary Report, 51 Ecology, 5, 802-809 (1970). Disponible en: http://www.jstor.org/stable/1933972. Hiroshi Iizuka, Hiroshi Okazaki \& Naosuke Seto, A New Sulfate-Reducing Bacterium Isolated from Antarctica, 15 Journal of General Applied Microbiology, 1, 11-18 (1969). Disponible en: https://www.jstage.jst.go.jp/article/jgam1955/15/1/15_1_11/_pdf. Margaret E. Di Menna, Yeast 
esto, la bioprospección solo empieza a ser considerada a finales de los noventa en el STA; en la academia, las obras que inician la discusión son las de John Bowman y David Nichols, en las cuales no se hace una exposición profunda de los aspectos jurídicos involucrados en la bioprospección antártica, sino que se analiza la importancia de esa actividad habida cuenta de la biodiversidad antártica, en particular por las condiciones ambientales en que se desarrolla la vida allín ${ }^{21}$.

Desde los autores citados hasta 2009, la literatura especializada estimó que el tema estaba en una fase exploratoria. Así se expresó en las Reuniones Consultivas del Tratado Antártico XXX, celebrada en 2007 New Delhi y en la XXXI, celebrada en 2008 en Kiev ${ }^{22}$. En 2009, se abrió una nueva etapa caracterizada por instrumentos para recabar y publicitar información referida a prospección biológica en la Antártida.

\section{La primera etapa del análisis jurídico de la bioprospección antártica}

En 2002, en el marco de la XXV RCTA, el Reino Unido presentó el WP-043 Bioprospecting in Antarctica (Bioprospección en la Antártida ${ }^{23}$ ), en el cual: (i) ante la indefinición sobre qué es la bio-

from Antarctica, 23 Journal of General Microbiology, 295-300 (1960). Disponible en: http:// mic.sgmjournals.org/content/23/2/295.full.pdf. Gordon DE QueTteville Robin, Research in Antarctica, 3-4 International Council for Science, ICSU Review, 337-350 (1961). MARY G. Rogick, Studies on Marine Bryozoa XIII. Tow New Genera and New Species from Antarctica, 119 Biological Bulletin, 3, 479-493 (1960). Disponible en: http://www.biolbull.org/content/119/3/479. full.pdf. Donald Eugene Wohlschlag, U.S. Biological Facilities in Antarctica, 15 BioScience, 4, 261-263 (1965).

21 John P. Bowman, Antarctica a Global "Hotspot": Biodiversity and Biotechnology, en Looking South-Managing Technology, Opportunities and The Global Environment, 85-90 (Australian Academy of Technological Sciences and Engineering, Parkville, 2002). David S. Nichols, Case Studies of Biotechnology Opportunities in Antarctica, en Looking South - Managing Technology, Opportunities and the Global Environment, 93-105 (Australian Academy of Technological Sciences and Engineering, Parkville, 2002).

22 Reunión Consultiva del Tratado Antártico XXX (New Delhi, 2007), Revisión, actualización y mecanismo propuesto para apoyar la acción futura en materia de prospección biológica en la Antártida, Information Paper 67, Agenda Item 17. Disponible en: http://www.ats.aq/devAS/ ats_meetings_documents.aspx?lang=s. Reunión Consultiva del Tratado Antártico XXXI (Kiev, 2008), Actualización sobre la bioprospección en la Antártida, incluida la creación de la Base de Datos sobre la Prospección Biológica en la Antártida, Working Paper 11, Agenda Item 17. Disponible en: http://www.ats.aq/devAS/ats_meetings_documents.aspx?lang=s

23 Reunión Consultiva del Tratado Antártico XXV (Varsovia, 2002), Bioprospecting in Antarctica, Working paper WP-043, Agenda Item CPA 4(d). Disponible en: http://www.ats.aq/devAS/ ats_meetings_documents.aspx?lang=s 
prospección, presentó una propuesta de qué debe entenderse por tal; (ii) resalta la falta de regulación sobre cómo deben repartirse las ganancias dado el régimen particular de la Antártida, pues la bioprospección es una actividad en la cual hay un gran interés por las ganancias que puede generar, y (iii) pone de manifiesto la incompatibilidad potencial entre la bioprospección y el principio de cooperación científica internacional consagrada en el artículo III del Tratado Antártico.

Tomando en cuenta estas consideraciones, al año siguiente se publicó un artículo clave para abordar jurídicamente el tema de la bioprospección antártica, al punto de que se ha convertido en una pieza fundamental para la discusión en la materia. Este artículo propone una conceptualización de bioprospección, y tres medidas para lograr una regulación de la bioprospección antártica: (i) que haya un pago a quien quiera realizarla por el acceso al material antártico, (ii) que las muestras permanezcan en un depósito común de conformidad con lo establecido en las Bonn Guidelines y (iii) que se cree un registro de marca y patentamiento para los productos provenientes de la Antártida ${ }^{24}$.

Por su parte, Dagmar Lohan y Sam Johnston ${ }^{25}$, del Instituto de Estudios Avanzados de la Universidad de las Naciones Unidas, se suman al debate sobre la bioprospección antártica, mediante un análisis de las tendencias de esa actividad y de las normas jurídicas internacionales que podrían aplicársele. Concluyen que la información sobre la bioprospección antártica presenta grandes vacíos, tendencia que debería revertirse para poder arribar a una definición que sea válida en el STA. Además, se debe determinar quién es el propietario de las muestras, cómo establecer un régimen de beneficios y si se contraría o no el contenido del artículo III del

24 Julia Jabour-Green \& Dianne Nicol, Bioprospecting in Areas Outside National Jurisdiction: Antarctica and the Southern Ocean, 4 Melbourne Journal of International Law, 76-111 (2003). Disponible en: http://eprints.utas.edu.au/2668/2/03Green-Nicol\%5B1\%5D.pdf. Bonn Guidelines on Access to Genetic Resources and Fair and Equitable Sharing of the Benefits Arising Out of Their Utilization, 2002. Disponible en: https://www.cbd.int/abs/bonn/

25 Sus estudios y propuestas se presentan en el STA, mediante el IP 075 en la XXVI RCTA (Madrid, 2003). Reunión Consultiva del Tratado Antártico XXVI (Madrid, 2003). Informe final. Disponible en: http://www.ats.aq/devAS/ats_meetings_meeting.aspx?lang=e. Reunión Consultiva del Tratado Antártico XXVI (Madrid, 2003), The International Regime for Bioprospecting: Existing Policies and Emerging Issues for Antarctica, Information Paper 075 UKI NO, Agenda Item VI CEP 7. Disponible en: http://www.ats.aq/devAS/ats_meetings_documents. aspx?lang=e 
$\mathrm{TA}^{26}$. Los mencionados autores retornan sobre estos mismos temas en un estudio posterior ${ }^{27}$.

Aprovechando la importancia que iba tomando la bioprospección antártica, tanto doctrinalmente como en las instituciones del STA, en el marco de la XXVI RCTA (Madrid, 2003), tuvo lugar el evento a partir del cual se han generado más aportes al debate teórico, denominado Bioprospecting in Antarctica. An Academic Workshop. Bajo la dirección de Michelle Rogan-Finnemore y Alan Hemmings, este encuentro abordó la bioprospección antártica desde tres dimensiones, a saber: (i) la ciencia y los intereses comerciales; (ii) el medio ambiente, la ética y la equidad; y, (iii) los aspectos legales. Bioprospecting in Antarctica. An Academic Workshop permitió en buena medida dar a conocer el tema y las múltiples dificultades que supone, dadas las regulaciones del STA ${ }^{28}$.

Con base en las experiencias obtenidas en el evento mencionado, Michelle Rogan-Finnemore planteó que en la bioprospección antártica hay dos temas fundamentales que se deben considerar para cualquier tipo de solución que se formule. Esos temas son la libre disponibilidad de información, consagrada en el artículo III TA, y el uso pacífico del territorio antártico, consagrado en el artículo I TA. En ese contexto, la autora concluye que la bioprospección tiene un impacto directo en las reclamaciones territoriales, razón por la cual la dinámica de las relaciones antárticas puede sufrir serios deterioros, pero en la medida en que haya una definición clara y técnica de la actividad de prospección biológica en la Antártida esos desafíos se pueden subsanar ${ }^{29}$.

Dadas las experiencias y el alcance del debate, Francia hizo una primera propuesta de regulación jurídica de la bioprospección

26 Sam Johnston \& Dagmar Lohan, The International Regime for Bioprospecting: Existing Policies and Emerging Issues for Antarctica, United Nations University - Institute of Advanced Studies, Yokohama (2003). Disponible en: http://i.unu.edu/media/unu.edu/publication/28671/ UNUIAS_AntarcticaReport-2.pdf

27 Dagmar Lohan \& Sam Johnston, Bioprospecting in Antarctica, 10, United Nations University - Institute of Advanced Studies, Yokohama (2005). Disponible en: http://i.unu.edu/media/unu. edu/publication/28551/antarctic_bioprospecting-3.pdf. Presentado al STA en el IP106 XXVII RCTA (Cape Town, 2004).

28 Michelle Rogan-Finnemore \& Alan Hemmings, eds., Bioprospecting in Antarctica, An Academic Workshop (Gateway Antarctica, University of Canterbury, Christchurch, New Zealand, 2003). Disponible en: http://www.anta.canterbury.ac.nz/resources/bio-prospecting/

29 Michelle Rogan-Finnemore, What Bioprospecting Means for Antarctica and the Southern Ocean, en International Law Issues in the South Pacific: Policies and Practices, 199-228 (Geoffrey W. G. Leane \& Barbara van Tigerstrom, eds., Ashgate Publishing Ltd., 2005). 
antártica en $2006^{30}$. Sin embargo, esta propuesta no ha tenido la recepción que debería y no ha trascendido mayormente. En ese año, en Chile, de cara a la formulación de una política antártica que aborde los aspectos más conflictivos de la realidad de las relaciones antárticas, María Teresa Infante-Caffi formuló que la bioprospección antártica representa un alto interés científico y económico, asunto que pone en juego varios aspectos como lo concerniente a los efectos de esta actividad en el ecosistema antártico, la libertad de investigación científica, las reglas de protección de la propiedad intelectual relativas a la generación de conocimiento y el dominio de técnicas aplicadas al material genético, a partir de su cosecha o recolección ${ }^{31}$. El STA aún no ha implementado en su totalidad estos temas.

Por otra parte, bajo el amparo del Instituto de Estudios Avanzados de la Universidad de las Naciones Unidas, David Leary formuló una propuesta metodológicamente nueva, que consiste en comparar el régimen de bioprospección en el Ártico y en la Antártida. En cuanto a la bioprospección antártica, expresa que la regulación del acceso al material biológico, la distribución de beneficios, la propiedad intelectual y la pugna entre la confidencialidad comercial y el principio de libre intercambio de información son los desafíos que deben ser tratados y solucionados por el STA, desde una perspectiva ética que equilibre la ciencia y la comercialización ${ }^{32}$.

En suma, la primera etapa del análisis sobre el régimen regulador de la bioprospección antártica deja en claro la complejidad del problema y el difícil balance entre comercio, ciencia, ética, reclamaciones territoriales, patrimonio de la humanidad y protección ambiental antártica. Es tan difícil afirmar sin críticas que se tiene derecho a explotar sin ninguna compensación, como no explotar e impedir el mejoramiento de la calidad de vida que se podría lograr con los resultados de la bioprospección antártica. Si bien la segunda

30 Reunión Consultiva del Tratado Antártico XXIX (Edinburgh, 2006), Informe de un taller sobre especies no autóctonas en la Antártida, Working Paper 13, Agenda Item ATCM 8. Disponible en: http://www.ats.aq/devAS/ats_meetings_documents.aspx?lang=s

31 María Teresa Infante-CAFFI, La política antártica chilena: nuevas realidades, 39 Revista de Estudios Internacionales, 155, 37-51 (2006). Disponible en: http://www.revistaei.uchile.cl/index. $\mathrm{php/REI/article/viewFile/14341/14657}$

32 DAVID LEARY, Bi-polar Disorder? Is Bioprospecting an Emerging Issue for the Arctic as well as for Antarctica?, 17 Review of European Community \& International Environmental Law, 1, 45-55 (2008). 
etapa debería haber logrado avances concretos, los anhelos superan la realidad, como se verá en las líneas posteriores.

\section{La segunda etapa del análisis jurídico de la bioprospección antártica}

Identificados los temas más conflictivos, a partir de 2009 hubo un nuevo impulso para abordar la problemática de la bioprospección antártica. En ese sentido, Karen Nadine Scott planteó la necesidad de crear una Autoridad Administrativa Antártica, que emule la Autoridad Internacional de los Fondos Marinos (International Seabed Authority, ISA), que tendría a su cargo la regulación de la propiedad de las muestras, la propiedad intelectual e industrial y el reparto de los beneficios ${ }^{33}$. A una solución muy similar arribó Jason Michael Davis, quien también considera que la forma más adecuada de abordar la gobernabilidad antártica es mediante un organismo especializado que se encargue de los asuntos relativos a la bioprospección ${ }^{34}$.

A estas propuestas se sumó Ann-Isabelle Guyomard, quien plantea como posible solución a los desafíos que impone la actividad de prospección biológica, la creación de un régimen antártico ad hoc, pues hay temas de extrema sensibilidad, como el reparto de beneficios, respecto de los cuales debe haber reglas precisas y con$\operatorname{cretas}^{35}$. A esto respondieron Kevin A. Hughes y Paul D. Bridge, que piden que antes de tomar una decisión se debata aún más, en especial en el seno del SCAR ${ }^{36}$.

Mientras el debate y las propuestas sobre el régimen antártico ad hoc para regular la bioprospección antártica se concretan en la

33 Karen N. Scott, Managing Sovereignty and Jurisdictional Disputes in the Antarctic, Conferencia del 20 Aniversario de la Unidad de Investigación de Fronteras Internacionales, celebrada en Durham, Reino Unido entre el 1 y el 3 de abril de 2009. Disponible en: https://www.dur. ac.uk/resources/ibru/conferences/sos/karen_scott_paper.pdf

34 Jason Michael Davis, Reconsidering Antarctic Bioprospecting through Territorialities of Science, Property and Governance (Tesis Doctoral, Ohio University, 2011). Disponible en: https://etd. ohiolink.edu/ap/10?0::NO:10:P10_ACCESSION_NUM:osu1299535648

35 Ann-Isabelle Guyomard, Ethics and Bioprospecting in Antarctica, 10 Ethics in Science and Environmental Politics, 31-44 (2010). Disponible en: http://www.int-res.com/articles/esep2010/10/ e010p031.pdf

36 Kevin A. Hughes \& Paul D. Bridge, Potential Impacts of Antarctic Bioprospecting and Associated Commercial Activities upon Antarctic Science and Scientists, 10 Ethics in Science and Environmental Politics, 13-18 (2010). Disponible en: http://www.int-res.com/articles/ esep2010/10/e010p013.pdf 
realidad, Julia Jabour planteó que sobre la Antártida hay varias visiones: una según la cual es un lugar común de la humanidad (global commons), como sostiene Malasia; otra en la cual es un patrimonio común de la humanidad, defendida por India en su momento; y la que defienden los países parte del STA, según la cual es una zona sui géneris. En el sentir de la autora, cada una de estas visiones lleva a formulaciones diferentes de regímenes reguladores de la bioprospección antártica, asunto que se agrava porque en el STA no hay uniformidad para delimitar el alcance de la Antártida, en especial en el ámbito ambiental y biológico. Dado lo anterior, Jabour propuso que mientras se llega a soluciones definitivas, el STA cobre a los bioprospectores, para que puedan realizar la actividad, y con estos recursos se cree un fondo común de apoyo a la ciencia antártica ${ }^{37}$.

Morten Walløe Tvedt reconoce las dificultades de aplicar el Convenio sobre la Diversidad Biológica a la bioprospección antártica, que es una de las posibles soluciones, y ante la falta de claridad que genera el TA en materia de jurisdicción sobre la propiedad de los recursos genéticos y biológicos, considera que el Derecho de Patentes es la herramienta más útil para crear derechos de exclusividad sobre estos. Para Tvedt, si el resultado de la investigación científica genera un producto nuevo, no obvio y susceptible de aplicación industrial, es decir, cumple los requisitos de patentabilidad, debe patentarse y en caso de contradicción entre el TA y el Derecho de Patentes, este último debe prevalecer, pues la patente no genera un derecho de propiedad sobre los recursos antárticos, sino un privilegio para que terceros no exploten la invención sin el consentimiento de quien obtuvo la patente.

Adicionalmente, para Tvedt, el principio de cooperación internacional en la investigación científica de la Antártida, que sería un obstáculo para la patentabilidad de la invención, no es un inconveniente por cuanto la frase "observaciones y resultados científicos" contenida en el artículo IIIc del Tratado Antártico no se define con precisión y por lo tanto, el espectro de esa obligación también es

37 Julia Jabour, Biological Prospecting: the Ethics of Exclusive Reward from Antarctic Activity, 10 Ethics in Science and Environmental Politics, 19-29 (2010). Disponible en: http://www.int-res. com/articles/esep2010/10/e010p019.pdf 
impreciso, de manera que esta obligación no alteraría la patentabilidad de una investigación científica antártica ${ }^{38}$.

Como es de suponer, la postura de Tvedt genera un gran desafío para el STA, pues si bien jurídicamente lo propuesto por él puede ser una solución válida, en lo político crea situaciones muy complejas y difíciles de resolver. En una línea más temperada que la de Tvedt, Hemmings considera que la bioprospección antártica puede realizarse y en caso de conflicto hay una serie de prioridades a examinar para resolverlo. Esa serie de prioridades tendría el siguiente orden: (i) las oportunidades económicas nacionales; (ii) la consistencia con el TA y otras normas de los compromisos antárticos; (iii) el estado de desarrollo de la política de bioprospección nacional; (iv) la cuestión de la creación precedente, que puede tener consecuencias en otros foros internacionales; (v) las implicaciones de cualquier evolución de la normativa internacional alrededor de la bioprospección (fuera del STA) en los foros internacionales, que también pueda aplicarse en el área del TA ${ }^{39}$.

Por su parte, Sanjay Chaturvedi sostiene que estas posiciones pueden ser muy adecuadas e incluso tienen un sustento en lo discutido en la RCTA XXVIII en el WP13, presentado por Nueva Zelanda y Suecia $^{40}$, según el cual la bioprospección no es inconsistente con el artículo III del Tratado Antártico; sin embargo, el mencionado autor aporta una pregunta muy importante en relación con la legalidad o no de la bioprospección antártica frente al TA, y que consiste en cuestionarse si las prácticas de las empresas que se dediquen a la bioprospección antártica se pueden sujetar a los principios y prácticas de transparencia fijadas en el TA, o si aplica la cultura del secreto, propia de las inversiones comerciales. Este cuestionamiento es fundamental para cualquier decisión que se tome en materia de bioprospección antártica, pues la mayoría de las inversiones no proviene de Estados sino de empresas privadas,

38 Morten Walløe Tvedt, Patent Law and Bioprospecting in Antarctica, 47 Polar Record, 1, 46-55 (2011). Disponible en: http://www.fni.no/publ/polar\&russia.html\#antarctic_bioprospecting, http://www.fni.no/doc\&pdf/MWT-PR-2010.pdf

39 Alan D. Hemmings, Does Bioprospecting Risk Moral Hazard for Science on the Antarctic Treaty System? 10 Ethics in Science and Environmental Politics, 5-12 (2010). Disponible en: http://www.int-res.com/articles/esep2010/10/e010p005.pdf

40 Reunión Consultiva del Tratado Antártico XXVIII (Stockholm, 2005), La prospección biológica en la Antártida. Working Paper 13, Agenda Item ATCM18. Disponible en: http://www. ats.aq/devAS/ats_meetings_documents.aspx?lang=e 
que tienen la capacidad financiera para realizar esas actividades y, por ende, debería crearse un régimen jurídico especial ${ }^{41}$.

Con base en la experiencia recogida desde el inicio de la discusión teórica y con una visión suramericana, Fernando Villamizar analiza el régimen jurídico aplicable a la bioprospección antártica y resalta las diversas indefiniciones e incompatibilidades jurídicas que se presentan en la práctica de esa actividad. Respecto de las indefiniciones, el aspecto más relevante apunta a preguntar que si el STA no propone definiciones, ¿cómo este va a regular la bioprospección? En cuanto a las incompatibilidades, destaca que varias de las etapas propias de la bioprospección antártica contrarían diversos principios y preceptos del STA. En razón de lo anterior, el autor plantea la necesidad de considerar un régimen particular para la bioprospección antártica basado en los propios planteamientos del STA y sin acudir a importar figuras jurídicas de otros ordenamientos normativos ${ }^{42}$.

A partir de la lectura de los diversos aportes que pretenden regular de manera adecuada la bioprospección antártica, se puede apreciar una relativa coincidencia en cuanto a la generación de una institucionalidad propia para la regulación de la actividad. En el siguiente acápite se expondrán algunos de los aún incipientes avances en esa materia.

\section{INCIPIENTE INSTITUCIONALIDAD DE LA BIOPROSPECCIÓN ANTÁRTICA DEL SISTEMA DEL TRATADO ANTÁRTICO}

Como se expresó anteriormente, el STA ha pretendido que la bioprospección antártica sea una práctica regulada por su propia institucionalidad. Sin embargo, la incipiente institucionalidad reguladora de la bioprospección antártica es fruto de esfuerzos de instituciones que si bien están ligadas al STA, no son parte del organismo. Pese a esto, lo relevante es que poco a poco se han ido

41 Sanjay Chaturvedi, Emerging Science - Geopolitics Interface in the Antarctic. The Indian Challenge, 16-31 (National Conference on Science \& Geopolitics of Arctic \& Antarctic, SaGAA, India, 2011). Disponible en: http://www.lights.org.in/sagaa/pdf_files/book.pdf

42 Fernando Villamizar-Lamus, La bioprospección antártica: indefiniciones e incompatibilidades con el Sistema del Tratado Antártico, 23 International Law, Revista Colombiana de Derecho Internacional, 53-86 (2013). Disponible en: http://www.scielo.org.co/pdf/ilrdi/n23/n23a03.pdf 
generando mecanismos para intentar regular las actividades de bioprospección, aunque no sea de una manera integral sino parcelada.

\section{A. Proyecto Micromat}

En el marco del Programa IV de la Unión Europea, y con su financiamiento, se erigió el proyecto Biodiversity of Microbial Mats in Antarctica, conocido como Micromat, que tuvo lugar entre 1999 y 2001. Micromat fue una asociación industrial, académica e institucional ${ }^{43}$ que tenía como objetivo mejorar el conocimiento de la biodiversidad de las bacterias, protistas y hongos en los tapetes microbianos de la Antártida y probar esta biodiversidad para nuevos compuestos de potencial uso biotecnológico ${ }^{44}$.

La relevancia de este proyecto para la institucionalización de la bioprospección antártica radica en que fijó unas reglas interesantes para proyectos de esta índole, según las cuales las empresas privadas no podían apropiarse de las muestras tomadas, sino que estas permanecerían en poder de los laboratorios de las instituciones científicas. En caso de que alguna muestra fuese susceptible de comercialización por parte de alguna de las empresas privadas participantes, la compañía interesada debía solicitar la autorización del laboratorio donde residía la muestra y del Estado reclamante del sector de donde se extrajo ${ }^{45}$. Esta solución, pese a lo criticable que pueda ser, es relevante porque permite combinar el interés cien-

43 El proyecto estaba compuesto por distintos estamentos: (i) académico, (ii) industrial, (iii) centros de investigación y (iv) de apoyo logístico. En el estamento académico participaron la Universidad de Liège (Bélgica), Universidad de Gent (Bélgica), Universidad de Bordeaux (Francia) y Nottingham (Reino Unido). En el estamento industrial, había tres integrantes, Merck Sharp \& Dohme (España), Genencor International y Biosearch - ahora Vicuron(Italia). En cuanto a los centros de investigación participantes estaban Deutsche Sammlung von Mikroorganismen und Zellkulturen GmbH (DSMZ) y Rheinisch-Westfälisches Institut für Wasserforschung Gemeinnützige de Alemania (IWW); NERC British Antarctic Survey (BAS) (Reino Unido); y el Institute of Royal Netherlands Academy of Arts and Science (Holanda). El apoyo logístico estaba a cargo de Australia y Estados Unidos. Para profundizar el papel de cada participante en el proyecto puede consultarse, Reunión Consultiva del Tratado Antártico XXVII (Cape Town, 2004), Industry Involvement in Antarctic Bioprospecting. Information Paper 106 UNEP, Agenda Item CEP 7 and ATCM 17, 4 y ss. Disponible en: http:// www.unep.org/dewa/Portals/67/pdf/bioprospecting_final.pdf

44 http://www.nerc-bas.ac.uk/public/mlsd/micromat/

45 Fernando Villamizar-Lamus, La bioprospección antártica: indefiniciones e incompatibilidades con el Sistema del Tratado Antártico, 23 International Law, Revista Colombiana de Derecho Internacional, 53-86 (2013). Disponible en: http://www.scielo.org.co/pdf/ilrdi/n23/n23a03.pdf 
tífico con el comercial, sin dejar de lado la reclamación territorial realizada por ciertos Estados.

Además de esto, el reparto de beneficios que se den como resultado de la comercialización de la propiedad intelectual que genere la investigación, tendrá que incluir no solo a los miembros del proyecto, como es obvio, sino que también debe considerar al Estado reclamante de territorio antártico ${ }^{46}$ en donde se realizó la bioprospección, que para el caso sub examine fue Australia ${ }^{47}$.

Entonces, en la medida en que estas disposiciones se institucionalicen por lo menos se evitará que empresas privadas se apoderen de las muestras biológicas antárticas y se establecerá un reparto de beneficios que incluya al Estado reclamante, con lo cual una fuente potencial de conflictos y de vulneraciones al STA se disminuiría. Sin embargo, precisamente estos dos aspectos, y en particular la imposibilidad de apropiación de las muestras, han hecho que proyectos que se auguraban como exitosos, se estanquen o no se realicen, pues los inversionistas, que generalmente son grandes multinacionales, no están dispuestos a sufragar las cuantiosas sumas que supone la investigación antártica sin la posibilidad de hacerse con la propiedad de las muestras.

Lo anteriormente mencionado ocurrió con ColdZyme, un proyecto europeo posterior a Micromat muy publicitado en su momento. ColdZyme tenía como socio industrial a la multinacional Unilever, y era un proyecto de bioprospección antártica sobre bacterias con enzimas activas al frío (cold-active enzymes), que debía desarrollar varias etapas. Superada la primera etapa que consistía en recolectar las muestras, Unilever suspendió el financiamiento ${ }^{48}$,

46 Los siete estados que han reclamado soberanía sobre territorios ubicados en la Antártida son Gran Bretaña, Nueva Zelanda, Francia, Australia, Noruega, Chile y Argentina.

47 Michelle Rogan-Finnemore \& Alan Hemmings, eds., Bioprospecting in Antarctica, An Academic Workshop, 6 (Gateway Antarctica, University of Canterbury, Christchurch, New Zealand, 2003). Disponible en: http://www.anta.canterbury.ac.nz/resources/bio-prospecting/

48 Diversos documentos del STA certifican este tipo de actitudes de las multinacionales. Entre otros, se puede consultar la Reunión Consultiva del Tratado Antártico XXVI (Madrid, 2003). Informe final. Disponible en: http://www.ats.aq/devAS/ats_meetings_meeting.aspx?lang=e. Reunión Consultiva del Tratado Antártico XXVI (Madrid, 2003), The International Regime for Bioprospecting: Existing Policies and Emerging Issues for Antarctica, Information Paper 075 UK/NO, Agenda Item VI CEP 7. Disponible en: http://www.ats.aq/devAS/ats_meetings_documents.aspx?lang=e 
pues la multinacional no tenía interés en continuar adelante si no podía apropiarse de las muestras de los microrganismos antárticos ${ }^{49}$.

Este desistimiento en la financiación de proyectos de bioprospección antártica puede ser visto como negativo ${ }^{50}$, en la medida en que la falta de recursos financieros puede desalentar la investigación científica y desvanecer los beneficios que para la humanidad tendrían los resultados de la bioprospección. Sin embargo, desde otro punto de vista, lo valioso del proyecto Micromat es que al establecer esas reglas, evita que las muestras biológicas antárticas sean apropiadas por empresas que financian un determinado proyecto, con lo cual se garantiza la biodiversidad antártica y se evitan prácticas que puedan ser consideradas como biopiratería ${ }^{51}$; en la medida en que las regulaciones del proyecto Micromat hagan carrera, se evitarán prácticas éticamente cuestionables respecto de las muestras biológicas.

\section{B. Bases de datos sobre bioprospección antártica}

En la RCTA XXX (New Delhi, 2007), se concretó un aspecto fundamental que ya venía haciendo carrera en las anteriores RCTA en las que se trató el tema de la bioprospección antártica. Este tema hace referencia a algo evidente, pero que no se tenía formalmente

49 Dagmar Lohan \& Sam Johnston, Bioprospecting in Antarctica, 9, United Nations University - Institute of Advanced Studies, Yokohama (2005). Disponible en: http://i.unu.edu/media/ unu.edu/publication/28551/antarctic_bioprospecting-3.pdf

50 Reunión Consultiva del Tratado Antártico XXVII (Cape Town, 2004), Industry Involvement in Antarctic Bioprospecting. Information Paper 106 UNEP, Agenda Item CEP 7 and ATCM 17, 2. Disponible en: http://www.ats.aq/devAS/ats_meetings_documents.aspx?lang=e, http:// www.unep.org/dewa/Portals/67/pdf/bioprospecting_final.pdf

51 Ho define biopiratería como "la apropiación de conocimientos o recursos genéticos de la agricultura y de las comunidades indígenas por parte de individuos o instituciones, que buscan el control monopólico exclusivo (por medio de patentes o propiedad intelectual) sobre esos recursos o esos conocimientos". Cynthia M. Ho, Biopiracy and Beyond: A Consideration of Socio-Cultural Conflicts with Global Patent Policies, 39 University of Michigan Journal of Law Reform, 433-542, 450 (2006). Disponible en: http://lawecommons.luc.edu/cgi/viewcontent.cgi? article $=1034 \&$ context $=$ facpubs. Este ítem fue tema de preocupación en la Reunión Consultiva del Tratado Antártico XXIX (Edinburgh, 2006), Recent Trends in Biological Prospecting, Information Paper 116, Agenda Item ATCM 18. Disponible en: http://www.ats.aq/devAS/ ats_meetings_documents.aspx?lang=e. Sobre cómo algunas empresas se apropian de material biológico e incurren en prácticas susceptibles de ser catalogadas como "biopiratería", además del profundo estudio de Ho, ya citado, también puede consultar, entre otros: GUNTHER Teubner \& Peter Korth, Two Kinds of Legal Pluralism: Collision of Transnational Regimes in the Double Fragmentation of World Society, en Regime Interaction, in International Law: Facing Fragmentation, 23-54 (Margaret Young, ed., Oxford University Press, Oxford, 2012). Disponible en: http://papers.ssrn.com/sol3/papers.cfm?abstract_id=1416041 
hasta ese momento: información suficiente sobre las actividades de bioprospección. Así las cosas, varias instituciones estatales e intergubernamentales comprometieron los recursos financieros y logísticos para crear bases de datos que fueran libremente disponibles.

Además, se hicieron compromisos interinstitucionales de colaboración y cooperación para conseguir y difundir información sobre la biodiversidad y la bioprospección en la Antártida. La idea es que la información de los diversos proyectos que tuvieron lugar con esta iniciativa permita el rastreo de la situación y con esto se puedan tomar decisiones más acertadas ${ }^{52}$. Como consecuencia de estas iniciativas, hay algunas bases de datos que son fuentes de información, pese a que hay exhortaciones para que el proceso mejore aún más la calidad de la información ${ }^{53}$, entre las más relevantes están:

1. WWW.BIOPROSPECTOR.ORG: esta fuente de información corresponde a la Iniciativa de Biodiplomacia de la Universidad de las Naciones Unidas y de su Instituto de Estudios Avanzados. Ha sido desarrollada y mantenida por esa institución académica, con el apoyo del Programa de las Naciones Unidas para el Medio Ambiente (PNUMA/UNEP) y el Ministerio de Energía, Medio Ambiente y Desarrollo Sostenible de Bélgica (Ministère de l'Energie, de l'Environnement et du Développement Durable, antiguo Ministerio Federal de Medio Ambiente). Su objetivo como herramienta de información es proporcionar detalles de la investigación y comercialización de productos que surgen a partir de muestras biológicas que proceden de actividades de bioprospección realizadas en zonas o espacios no sometidos a una jurisdicción nacional, en particular en el Ártico, los fondos oceánicos y la Antártida.

Gracias a esa información se puede evaluar y documentar el alcance de la prospección biológica en las zonas en las cuales se hace el registro, y entre las cuales está la Antártida. La base de datos es muy sencilla y amigable para hacer búsquedas, pues en la página de inicio se puede seleccionar la zona del mundo que se

52 Reunión Consultiva del Tratado Antártico XXX (New Delhi, 2007), Revisión, actualización y mecanismo propuesto para apoyar la acción futura en materia de prospección biológica en la Antártida, Information Paper 67, Agenda Item 17. Disponible en: http://www.ats.aq/devAS/ ats_meetings_documents.aspx?lang=s

53 Reunión Consultiva del Tratado Antártico XXXVI (Brussels, 2013), Biological Prospecting in Antarctica - the Need for Improved Information. Working Paper 48, Agenda Item 17. Disponible en: http://www.ats.aq/devAS/ats_meetings_documents.aspx?lang=e 
quiera revisar ${ }^{54}$; una vez seleccionada esta, se puede buscar la bioprospección por área de investigación, nombre de la organización o gobierno participante y, de esa manera, monitorear el avance de la bioprospección que se ha concretado en patentes de invención.

2. $W W W . B I O D I V E R S I T Y . A Q^{55}$ : es un proyecto financiado por la Oficina de Política Científica de Bélgica, que nace en el marco del Año Polar Internacional 2007-2008, en virtud de la variedad biológica de la Antártida que había sido estudiada pero cuya información tenía dificultades para ser difundida. Así las cosas, el objetivo de www.biodiversity.aq es generar un sistema de información sobre la biodiversidad de la Antártida, de conformidad con los principios de la Global Biodiversity Information Facility (GBIF), de la cual se dará cuenta más adelante. Esta página web es la herramienta de gestión de datos y repositorio para la investigación relacionada con la biodiversidad conducida en la Estación Princess Elisabeth, pero aparte de las publicaciones realizadas por el personal científico, que van más allá de las patentes, los usuarios pueden publicar información sobre biodiversidad antártica, con lo cual se marca una notoria diferencia con www.bioprospector.org.

\section{Herramientas que sirven de fuente para las bases de datos de bioprospección antártica}

Aunque estas herramientas informáticas no son parte de las iniciativas de la RCTA XXX, han sido instrumentos fundamentales para el avance del conocimiento de las actividades de bioprospección

54 Las zonas aparecen en inglés, que es el idioma empleado en esa página web: Antarctic, Marine, Pacific o Arctic.

55 Según lo menciona el WP37 presentado por el Comité Científico para la Investigación Antártica (SCAR) y Bélgica en la RCTA XXXVI (Bruselas, 2013), biodiversity.aq "compila y gestiona la información nueva y la que ya existe sobre la biodiversidad marina de la Antártida, coordinando, dando apoyo, completando y mejorando la red de trabajo de las bases de datos, centrándose especialmente en la interoperabilidad. Esta información está disponible para las iniciativas de información sobre biodiversidad global como Global Biodiversity Information Facility (GBIF: www.gbif.org), Ocean Biogeographic Information System (OBIS: www.iobis. org) o Encyclopedia of Life (www.eol.org/). La política de datos de Biodiversity.aq se alinea con los requisitos del Tratado Antártico, y el Año Polar Internacional 2007-2008, así como los estándares de datos y metadatos promocionados por GBIF, OBIS, el Comité Permanente sobre Gestión de Datos Antárticos de SCAR (SCADM) y el Directorio Maestro Antártico Global (GCMD)". Reunión Consultiva del Tratado Antártico XXXVI (Brussels, 2013), www. biodiversity. aq La nueva red de información sobre biodiversidad antártica. Working Paper 37, Agenda Item 11. Disponible en: http://www.ats.aq/devAS/ats_meetings_documents.aspx?lang $=\mathrm{s}$ 
antártica $^{56}$. La más relevante es Global Biodiversity Information Facility (GBIF). Este proyecto surgió de una recomendación formulada en 1999 por el Biodiversity Informatics Subgroup of the Megascience Forum, institución establecida por la Organización para la Cooperación y el Desarrollo Económicos (OCDE). Concretamente, en esa ocasión se recomendó el establecimiento de una Red Mundial de Información de la Biodiversidad, para permitir a los usuarios navegar y poder utilizar grandes cantidades de información sobre biodiversidad. Esa recomendación fue aprobada por los encargados estatales de ciencia de la OCDE en 2001, lo que dio el sustento jurídico para la creación de GBIF.

GBIF es una base de datos internacional abierta, financiada por aportes gubernamentales, que opera por medio de una red de nodos, con la coordinación de los servicios de información de la biodiversidad de los organismos y países participantes. GBIF ofrece acceso gratuito y descargas ilimitadas para todos los registros publicados en la red para su uso en investigación. Se puede explorar por acontecimientos, especies, conjuntos de datos y países. Para los estudios actuales de la realidad y las tendencias de bioprospección antártica, GBIF constituye una herramienta fundamental, junto con el Access and Benefit Sharing Patent Index (ABSPAT), que es menos relevante que GBIF. ABSPAT es una herramienta que contiene la documentación de once millones de patentes provenientes de seis millones de especies y su acceso es restringido.

\section{Depósito o banco de muestras biológicas (ex situ collections)}

Como actividad científica, para su realización, la bioprospección requiere el agotamiento de varias etapas. La primera etapa es la recolección de muestras. La segunda etapa corresponde al aislamiento, caracterización y cultivo de las especies. La tercera etapa consiste en detectar la utilidad de las muestras para fines comerciales. La cuarta etapa corresponde al desarrollo, patentabilidad,

56 Un ejemplo de esto se puede constatar en la Reunión Consultiva del Tratado Antártico XXXVI (Brussels, 2013), An Update on Status and Trends Biological Prospecting in Antarctica and Recent Policy Developments at the International Level. Information Paper 22, Agenda Item 17. Disponible en: http://www.ats.aq/devAS/ats_meetings_documents.aspx?lang=s 
venta y marketing del producto ${ }^{57}$. Una vez finalizadas estas etapas, uno de los puntos más difíciles, y de los cuales ya se pudo entrever cuando se trató el tema del proyecto Micromat, es qué hacer con las muestras biológicas tomadas para hacer la prospección biológica cuando el proyecto llega a su fin.

Para evitar el apoderamiento de las muestras biológicas por parte de particulares, se han ido creando o habilitando bancos de muestras biológicas antárticas (mejor conocidos como ex situ collections por su denominación en el STA). Infortunadamente, estos bancos de muestras no tienen una uniformidad en su conformación, de suerte que varían desde pequeños laboratorios de universidades, pasando por colecciones estatales, hasta grandes colecciones comerciales. Es de esperar que iniciativas como la Task Force on Biological Resource Centres, propuesta por la Organización para la Cooperación y el Desarrollo Económicos (OCDE) ${ }^{58}$, ayuden a homogeneizar la conformación, organización y tamaño de los bancos de muestras para el tema antártico.

Un rastreo hecho por Bélgica y la Universidad de las Naciones Unidas - Instituto de Estudios Avanzados da cuenta de 23 bancos de muestras biológicas antárticas, una cifra alentadora por la cantidad de entidades comprometidas a preservar la diversidad biológica y genética de la Antártida. Dado que esa cifra es referencial ${ }^{59}$, es de esperar que esos bancos de muestras sean los más relevantes, y que haya más. Los bancos de muestras biológicas antárticas detectados son:

57 Julia Jabour-Green \& Dianne Nicol, Bioprospecting in Areas Outside National Jurisdiction: Antarctica and the Southern Ocean, 4 Melbourne Journal of International Law, 76-111, 85 (2003). Disponible en: http://eprints.utas.edu.au/2668/2/03Green-Nicol\%5B1\%5D.pdf

58 Para profundizar sobre las iniciativas de la OCDE en esta materia, se puede consultar entre otros reportes: Organisation for Economic Co-operation and Development, OECD, Biological Resource Centres. Underpinning the Future of Life Sciences and Biotechnology (OECD Publishing, Paris, 2001). Disponible en: http://www.oecd.org/sti/biotech/2487422.pdf

59 Reunión Consultiva del Tratado Antártico XXXIII (Punta del Este, 2010), The Role of Ex-Situ Collections in Antarctic Bioprospecting, Information Paper 96, Agenda Item ATCM 17, 5. Disponible en. http://www.ats.aq/devAS/ats_meetings_documents.aspx?lang=e 


\section{Tabla 1}

\begin{tabular}{|c|c|c|}
\hline Estado & Nombre del banco de muestras & Sitio web \\
\hline Alemania & $\begin{array}{l}\text { German Collection of Microorganisms and } \\
\text { Cell Cultures (DSMZ) }\end{array}$ & $\begin{array}{l}\text { http://www.dsmz.de/ } \\
\text { index.htm }\end{array}$ \\
\hline Australia & $\begin{array}{l}\text { Australian Collection of Antarctic } \\
\text { Microorganisms (ACAM) }\end{array}$ & $\begin{array}{l}\text { http://www.antcrc.utas. } \\
\text { edu.au/antcrc/micropro/ } \\
\text { acaminfo }\end{array}$ \\
\hline Australia & $\begin{array}{l}\text { CSIRO [Commonwealth Scientific and } \\
\text { Industrial Research Organisation] Collection } \\
\text { of Living Microalgae }\end{array}$ & $\begin{array}{l}\text { http://www.marine.csiro. } \\
\text { au/algaedb/default.htm }\end{array}$ \\
\hline Bélgica & $\begin{array}{l}\text { Belgian Coordinated Collections of } \\
\text { Microorganisms }\end{array}$ & $\begin{array}{l}\text { http://bccm. } \\
\text { belspo.be/projects/ } \\
\text { programme2005-2008/ } \\
\text { c30014/ }\end{array}$ \\
\hline Brasil & $\begin{array}{l}\text { Brazilian Collection of Microorganisms } \\
\text { from the Environment and Industry (Coleção } \\
\text { Brasileira de Micro-organismos de Ambiente e } \\
\text { Indústria-CBMAI) }\end{array}$ & $\begin{array}{l}\text { http://webdrm.cpqba. } \\
\text { unicamp.br/ }\end{array}$ \\
\hline $\begin{array}{l}\text { Corea del } \\
\text { Sur }\end{array}$ & $\begin{array}{l}\text { Korean Agricultural Culture Collection } \\
\text { (KACC) }\end{array}$ & http://kacc.rda.go.kr \\
\hline $\begin{array}{l}\text { Estados } \\
\text { Unidos }\end{array}$ & American Type Culture Collection (ATCC) & http://www.atcc.org/ \\
\hline $\begin{array}{l}\text { Estados } \\
\text { Unidos }\end{array}$ & Antarctic Protist Culture Collection & $\begin{array}{l}\text { http://www.whoi.edu/ } \\
\text { science/B/protists/ }\end{array}$ \\
\hline $\begin{array}{l}\text { Estados } \\
\text { Unidos }\end{array}$ & Verenium & $\begin{array}{l}\text { http://www.verenium. } \\
\text { com/ }\end{array}$ \\
\hline $\begin{array}{l}\text { Estados } \\
\text { Unidos }\end{array}$ & Magellan BioSciences & $\begin{array}{l}\text { http://www. } \\
\text { magellanbioscience.com/ } \\
\text { libraries/index.html }\end{array}$ \\
\hline Finlandia & $\begin{array}{l}\text { VTT [Valtion Teknillinen Tutkimuskeskus] } \\
\text { Culture Collection }\end{array}$ & $\begin{array}{l}\text { http://culturecollection. } \\
\text { vtt.fi/ }\end{array}$ \\
\hline Holanda & $\begin{array}{l}\text { Centraalbureau voor Schimmelcultures (CBS) } \\
\text { Fungal Biodiversity Centre }\end{array}$ & http://www.cbs.knaw.nl \\
\hline India & $\begin{array}{l}\text { Microbial Type Culture Collection \& Gene } \\
\text { Bank (MTCC) }\end{array}$ & $\begin{array}{l}\text { http://www.imtech.res. } \\
\text { in/mtcc/ }\end{array}$ \\
\hline Japón & Japan Collection of Microorganisms & http://www.jcm.riken.jp \\
\hline Malasia & $\begin{array}{l}\text { University of Malaya Algae Culture Collection } \\
\text { (UMACC) }\end{array}$ & $\begin{array}{l}\text { http://sciencelinks.jp/ } \\
\text { j-east/article/ } \\
\text { 200310/000020031003 } \\
\text { A0266228.php }\end{array}$ \\
\hline $\begin{array}{l}\text { Nueva } \\
\text { Zelanda }\end{array}$ & $\begin{array}{l}\text { University of Waikato Antarctic Culture } \\
\text { Collection }\end{array}$ & $\begin{array}{l}\text { http://adt.waikato. } \\
\text { ac.nz/public/adt- } \\
\text { uow20070501.175007/ } \\
\text { index.html }\end{array}$ \\
\hline
\end{tabular}




\begin{tabular}{|l|l|l|}
\hline \multicolumn{1}{|c|}{ Estado } & \multicolumn{1}{|c|}{ Nombre del banco de muestras } & \multicolumn{1}{c|}{ Sitio web } \\
\hline $\begin{array}{l}\text { Nueva } \\
\text { Zelanda }\end{array}$ & ZyGEM & $\begin{array}{l}\text { http://www.Zygem. } \\
\text { com/Technology/Tech- } \\
\text { Culture-Collection.html }\end{array}$ \\
\hline Noruega & $\begin{array}{l}\text { Norwegian Institute for Water Research } \\
\text { (Norsk Institutt for Vannforskning, NIVA) }\end{array}$ & $\begin{array}{l}\text { http://www.niva.no/en/ } \\
\text { index }\end{array}$ \\
\hline $\begin{array}{l}\text { Reino } \\
\text { Unido }\end{array}$ & Culture Collection of Algae and Protozoa & http://www.ccap.ac.uk/ \\
\hline $\begin{array}{l}\text { Reino } \\
\text { Unido }\end{array}$ & British Antarctic Survey (BAS) Herbarium & $\begin{array}{l}\text { http://www.antarctica. } \\
\text { ac.uk//bas_research/ } \\
\text { data/collections/plant. } \\
\text { php) }\end{array}$ \\
\hline $\begin{array}{l}\text { Reino } \\
\text { Unido }\end{array}$ & CABI Genetic Resource Collection & $\begin{array}{l}\text { http://194.203.77.76/grc/ } \\
\text { index.htm http://www. } \\
\text { cabi.org/ }\end{array}$ \\
\hline $\begin{array}{l}\text { República } \\
\text { Checa }\end{array}$ & Culture Collection of Autotrophic Organisms & $\begin{array}{l}\text { http://www.butbn.cas.cz/ } \\
\text { ccala/index.php }\end{array}$ \\
\hline Rusia & VKM [Vseros Kollektsiya Mikroorganizmov] & http://www.vkm.ru/ \\
\hline
\end{tabular}

Fuente: elaboración propia con base en Reunión Consultiva del Tratado Antártico XXXIII (Punta del Este, 2010), The Role of Ex-Situ Collections in Antarctic Bioprospecting, Information Paper 96, Agenda Item ATCM 17. Disponible en. http://www.ats.aq/devAS/ats_meetings_documents. aspx?lang=e

\section{E. Presentaciones de actividades de los Estados que rea- lizan bioprospección antártica}

Recientemente, de manera unilateral, varios Estados han expuesto en el marco de las RCTA las actividades que realizan en materia de bioprospección antártica. En esta práctica se destacan países como Argentina, Bélgica ${ }^{60}$, Brasil ${ }^{61}$, Ecuador ${ }^{62}$ y Rumania ${ }^{63}$, y es una medida muy sana puesto que hace transparente qué hace cada

60 Reunión Consultiva del Tratado Antártico XXXV (Hobart, 2012), Report on the Bioprospecting Activities Carried Out by Belgian Scientists since 1998, Information Paper 22, Agenda Item 18. Disponible en: http://www.ats.aq/devAS/ats_meetings_documents.aspx?lang=e

61 Reunión Consultiva del Tratado Antártico XXXII (Baltimore, 2009), Bioprospecting Activities of Brazil in Antarctica: A Short Report, Information Paper 115, Agenda Item 17. Disponible en: http://www.ats.aq/devAS/ats_meetings_documents.aspx?lang=e

62 Reunión Consultiva del Tratado Antártico XXXIII (Punta del Este 2010), Informe de proyectos de bioprospección impulsados por el Ecuador, 2009-2010, Information Paper 125, Agenda Item 17. Disponible en: http://www.ats.aq/devAS/ats_meetings_documents.aspx?lang=e

63 Reunión Consultiva del Tratado Antártico XXXV (Hobart, 2012), Management Plan for Romanian Biological Prospecting Activities in Antarctica, Information Paper 84, Agenda Item 18. Disponible en: http://www.ats.aq/devAS/ats_meetings_documents.aspx?lang=e 
Estado en esta actividad. Tal vez el caso más destacado es el de Argentina que, desde 2006 y con cierta recurrencia, hace públicas las actividades de bioprospección antártica que realiza, con lo cual es el Estado que más informa sobre el particular ${ }^{64}$.

Esta práctica es tan positiva que la XXXVI RCTA, Bruselas 2013, promulgó la Resolución 6 (2013), que recomienda a los gobiernos informar sobre las actividades de prospección biológica que realizan "para facilitar una mejor comprensión y evaluación de este tipo de actividades" y examinar las formas de mejorar el intercambio de información al respecto. Se espera que a partir de la mencionada Resolución todos los Estados que hagan bioprospección antártica acaten esta disposición, con lo cual se mejorará la información y se podrán tomar políticas que aborden mejor la problemática y los desafíos que esta actividad implica.

64 Reunión Consultiva del Tratado Antártico XXIX (Edinburgh, 2006), Argentine Activities of Bioprospecting and Bioremediation in Antarctica, Information Paper 112, Agenda Item ATCM 18. Disponible en: http://www.ats.aq/devAS/ats_meetings_documents.aspx?lang=e. Reunión Consultiva del Tratado Antártico XXXII (Baltimore, 2009), Update of the Activities of the Argentine Antarctic Program on Bioprospection and Bioremediation in the Antarctica, Information Paper 84, Agenda Item 17. Disponible en: http://www.ats.aq/devAS/ats_meetings_documents. aspx?lang=e. Reunión Consultiva del Tratado Antártico XXXIV (Buenos Aires, 2011), Informe sobre las recientes actividades de bioprospección desarrolladas por Argentina durante el período 2010-2011, Information Paper 16, Agenda Item 17. Disponible en: http://www.ats.aq/devAS/ ats_meetings_documents.aspx?lang=e 


\section{CONCLUSIONES}

La bioprospección antártica como fenómeno relativamente nuevo genera muchos desafíos, dadas las características especiales que regulan jurídicamente la Antártida, en virtud de lo establecido en el STA. Es fundamental estudiar de manera muy seria la gran variedad de aristas e intereses contrapuestos, pues es de toda lógica que el continente blanco debe seguir siendo un territorio que se utilice exclusivamente para fines pacíficos, tal como lo fija el artículo I del TA. En ese estudio ha habido múltiples propuestas por parte de la Academia, que en alguna medida han hecho carrera y han sido presentadas en el STA. Sin embargo, los desafíos son tales que aún falta mucho camino por recorrer tanto en el campo académico como en el institucional antártico.

Como se ha podido apreciar con esta exposición, es muy notorio que se está generando paulatinamente una institucionalidad reguladora de la bioprospección antártica, que tiene como punto de partida canalizar la información sobre esa actividad para poder tomar decisiones más profundas. También es notorio que esa institucionalidad todavía es muy débil e incipiente y no tiene la obligatoriedad jurídica requerida, dados todos los intereses que hay en juego, salvo en lo concerniente a las presentaciones individuales de los Estados establecida en la Resolución 6 (2013), lo cual se ajusta en buena proporción a la hipótesis de trabajo de este artículo.

Dadas las características particulares de la Antártida, pensar en desaprovechar las riquezas biológicas que pudieran contribuir a una mejor calidad de vida de los habitantes de la Tierra es indeseable, pero tampoco lo es que unos entes privados se apropien de esas riquezas naturales de manera tal que incurran en biopiratería. Además, no se puede hacer caso omiso a las reclamaciones territoriales, ni al reparto de beneficios que debería proceder ante un proyecto exitoso de bioprospección antártica. Todos estos intereses hacen que no sea fácil establecer una regulación y que los pasos hacia una institucionalización sean más lentos de lo esperable, lo cual no deja de ser inquietante en la medida en que, sin una institucionalidad clara y sólida, el fenómeno se pueda desbordar y generar grietas en un esquema relativamente exitoso como lo ha sido el STA. 
Comoquiera que el principio de libertad de investigación científica consagrado en los artículos II y III del TA permite que cualquiera pueda investigar en la Antártica, por supuesto sujetándose a las regulaciones del STA, lo ideal sería que existiera un solo estatuto vinculante no solo para todos los Estados parte del TA, sean miembros consultivos o no, y para todo aquel interesado en realizar investigación científica y en particular bioprospección antártica, que regulara de manera integral los álgidos aspectos que implica la práctica de la prospección biológica. Sin embargo, lo que está aconteciendo es la creación de instituciones reguladoras que surgen poco a poco y de forma poco integrada, que además no resuelven temas tan candentes como el reparto de beneficios.

Visto lo anterior, se puede concluir que estamos en presencia de una regulación sui géneris de la bioprospección antártica, que obedece de cierta manera también a la naturaleza sui géneris y flexible del STA. Ojalá que esa flexibilidad y la voluntad de los Estados parte arriben pronto a una institucionalidad fuerte que evite exabruptos y situaciones anómalas que pongan en riesgo el hasta ahora exitoso STA. La doctrina se ha preocupado por formular múltiples propuestas encaminadas a regular esta materia; sin embargo, pocas se han llevado a cabo. Es decir, hay ideas para regular la bioprospección antártica, la pregunta entonces es: ¿̧habrá la voluntad política para llevarlas a cabo? 


\section{BIBLIOGRAFÍA}

\section{Libros}

Organisation for Economic Co-operation and Development, OECD, Biological Resource Centres. Underpinning the Future of Life Sciences and Biotechnology (OECD Publishing, Paris, 2001). Disponible en: http://www.oecd.org/sti/biotech/2487422. pdf

Rogan-Finnemore, Michelle \& Hemmings, Alan, eds., Bioprospecting in Antarctica, An Academic Workshop (Gateway Antarctica, University of Canterbury, Christchurch, New Zealand, 2003). Disponible en: http://www.anta.canterbury. ac.nz/resources/bio-prospecting/

\section{Contribuciones en obras colectivas}

Bowman, John P., Antarctica a Global "Hotspot": Biodiversity and Biotechnology, en Looking South - Managing Technology, Opportunities and The Global Environment, 85-90 (Australian Academy of Technological Sciences and Engineering, Parkville, 2002).

Chaturvedi, SAnjay, Emerging Science - Geopolitics Interface in the Antarctic. The Indian Challenge, 16-31 (National Conference on Science \& Geopolitics of Arctic \& Antarctic, SaGAA, India, 2011). Disponible en: http://www.lights.org.in/sagaa/ pdf_files/book.pdf

Kaye, Stuart; Johnson, Michael \& Baird, Rachel, Law, en Australia and the Antarctic Treaty System. 50 Years of Influence, 97-117 (Marcus HawArd \& TOM GrifFiths, eds., University of New South Wales Press Ltd., Sidney, 2011).

Nichols, David S., Case Studies of Biotechnology Opportunities in Antarctica, en Looking South-Managing Technology, Opportunities and the Global Environment, 93-105 (Australian Academy of Technological Sciences and Engineering, Parkville, 2002).

Rogan-Finnemore, Michelle, What Bioprospecting Means for Antarctica and the Southern Ocean, en International Law Issues in the South Pacific: Policies and Practices, 199-228 (Geoffrey W. G. Leane \& Barbara van Tigerstrom, eds., Ashgate Publishing Ltd., 2005).

Teubner, Gunther \& Korth, Peter, Two Kinds of Legal Pluralism: Collision of Transnational Regimes in the Double Fragmentation of World Society, en Regime Interaction, in International Law: Facing Fragmentation, 23-54 (MARGARET YounG, ed., Oxford University Press, Oxford, 2012). Disponible en: http://papers. ssrn.com/sol3/papers.cfm?abstract_id=1416041 


\section{Revistas}

Burkholder, Paul R. \& Mandelli, Enrique F., Carbon Assimilation of Marine Phytoplankton in Antarctica, 54 Botany, 437-444 (1965). Disponible en: http:// www.ncbi.nlm.nih.gov/pmc/articles/PMC219685/pdf/pnas00160-0103.pdf

Cameron, Alexander Scott, Staphylococcal Epidemiology in Antarctica, 68 The Journal of Hygiene, 1, 43-52 (1970). Disponible en: http://www.ncbi.nlm.nih.gov/pmc/ articles/PMC2130780/pdf/jhyg00091-0049.pdf

Cameron, Roy E. \& Benoit, Robert E., Microbial and Ecological Investigations of Recent Cinder Cones, Deception Island, Antarctica. A Preliminary Report, 51 Ecology, 5, 802-809 (1970). Disponible en: http://www.jstor.org/stable/1933972

Guyomard, Ann-Isabelle, Ethics and Bioprospecting in Antarctica, 10 Ethics in Science and Environmental Politics, 31-44 (2010). Disponible en: http://www.int-res.com/ articles/esep2010/10/e010p031.pdf

Hemmings, Alan D., Does Bioprospecting Risk Moral Hazard for Science on the Antarctic Treaty System? 10 Ethics in Science and Environmental Politics, 5-12 (2010). Disponible en: http://www.int-res.com/articles/esep2010/10/e010p005.pdf

Ho, Cynthia M., Biopiracy and Beyond: A Consideration of Socio-Cultural Conflicts with Global Patent Policies, 39 University of Michigan Journal of Law Reform, 433-542 (2006). Disponible en: http://lawecommons.luc.edu/cgi/viewcontent.cg i? article $=1034 \&$ contex $\mathrm{t}=$ facpubs

Hughes, Kevin A. \& Bridge, Paul D., Potential Impacts of Antarctic Bioprospecting and Associated Commercial Activities upon Antarctic Science and Scientists, 10 Ethics in Science and Environmental Politics, 13-18 (2010). Disponible en: http:// www.int-res.com/articles/esep2010/10/e010p013.pdf

Iizuka, Hiroshi; Okazaki, Hiroshi \& Seto, Naosuke, A New Sulfate-Reducing Bacterium Isolated from Antarctica, 15 Journal of General Applied Microbiology, 1, 11-18 (1969). Disponible en: https://www.jstage.jst.go.jp/article/jgam1955/15/1/15_1_11/_ pdf

INFANTE-CAFFI, MARía TeResa, La política antártica chilena: nuevas realidades, 39 Revista de Estudios Internacionales, 155, 37-51 (2006). Disponible en: http://www.revistaei. uchile.cl/index.php/REI/article/viewFile/14341/14657

JABour, Julia, Biological Prospecting: the Ethics of Exclusive Reward from Antarctic Activity, 10 Ethics in Science and Environmental Politics, 19-29 (2010). Disponible en: http://www.int-res.com/articles/esep2010/10/e010p019.pdf

Jabour-Green, Julia \& Nicol, Dianne, Bioprospecting in Areas Outside National Jurisdiction: Antarctica and the Southern Ocean, 4 Melbourne Journal of International Law, 76-111 (2003). Disponible en: http://eprints.utas.edu. au/2668/2/03Green-Nicol\%5B1\%5D.pdf

Leary, David, Bi-polar Disorder? Is Bioprospecting an Emerging Issue for the Arctic as well as for Antarctica?, 17 Review of European Community \& International Environmental Law, 1, $45-55$ (2008).

McLaughlin, Richard J., Foreign Access to Shared Marine Genetic Materials: Management Options for a Quasi-Fugacious Resource, 34 Ocean Development \& International Law, 3-4, 297-348 (2003). 
Menna, Margaret E. DI, Yeast from Antarctica, 23 Journal of General Microbiology, 295300 (1960). Disponible en: http://mic.sgmjournals.org/content/23/2/295.full.pdf

Robin, Gordon de Quetteville, Research in Antarctica, 3-4 International Council for Science, ICSU Review, 337-350 (1961).

Rogick, Mary G., Studies on Marine Bryozoa XIII. Tow New Genera and New Species from Antarctica, 119 Biological Bulletin, 3, 479-493 (1960). Disponible en: http:// www.biolbull.org/content/119/3/479.full.pdf

Schoenberg, Pamela L., A Polarizing Dilemma: Assessing Potential Regulatory GapFilling Measures for Arctic and Antarctic Marine Genetic Resource Access and Benefit Sharing, 42 International Law Journal, 2, 271-299 (2009). Disponible en: http://scholarship.law.cornell.edu/cgi/viewcontent.cgi?article=1748\&context=cilj

Tvedt, Morten Walløe, Patent Law and Bioprospecting in Antarctica, 47 Polar Record 1, $46-55$ (2011). Disponible en: http://www.fni.no/publ/polar\&russia. html\#antarctic_bioprospecting, http://www.fni.no/doc\&pdf/MWT-PR-2010.pdf

Villamizar-Lamus, Fernando, Del descubrimiento a la diplomacia: las etapas históricas de las relaciones antárticas, 10 Tradición y Saber, 2, 51-64 (2013). Disponible en: http://www.academia.edu/8595672/Del_descubrimiento_a_la_diplomacia_las_ etapas_de_las_relaciones_ant $\%$ C3\%Alrticas

Villamizar-Lamus, Fernando, La bioprospección antártica: indefiniciones e incompatibilidades con el Sistema del Tratado Antártico, 23 International Law, Revista Colombiana de Derecho Internacional, 53-86 (2013). Disponible en: http:// www.scielo.org.co/pdf/ilrdi/n23/n23a03.pdf

Villamizar-Lamus, Fernando, Tratado Antártico y Mecanismos de Protección del Territorio Antártico, 21 International Law, Revista Colombiana de Derecho Internacional, 255-295 (2012). Disponible en: http://www.redalyc.org/ pdf/824/82425523009.pdf

Weber, Melissa, Accreditation as a Regulatory Option for Antarctic Bioprospecting, 42 Polar Record, 4, 349-357 (2006).

Wohlschlag, Donald Eugene, U.S. Biological Facilities in Antarctica, 15 BioScience, 4, 261-263 (1965).

Zewers, Kirsten E., Bright Future for Marine Genetic Resources, Bleak Future for Settlement of Ownership Rights: Reflections on the United Nations Law of the Sea Consultative Process on Marine Genetic Resources, 5 Loyola University Chicago International Law Review, 2, 151-176 (2008). Disponible en: http://lawecommons. luc.edu/cgi/viewcontent.cgi?article $=1066 \&$ context $=$ lucilr

\section{Reportes}

Ernst \& Young, Beyond Borders: Global Biotechnology Report 2012. Disponible en: http://www.ey.com/GL/en/Industries/Life-Sciences/Beyond-borders---globalbiotechnology-report-2012

JohnSton, SAm \& Lohan, Dagmar, The International Regime for Bioprospecting: Existing Policies and Emerging Issues for Antarctica, United Nations University-Institute 
of Advanced Studies, Yokohama (2003). Disponible en: http://i.unu.edu/media/ unu.edu/publication/28671/UNUIAS_AntarcticaReport-2.pdf

Lohan, Dagmar \& Johnston, Sam, Bioprospecting in Antarctica, United Nations University - Institute of Advanced Studies, Yokohama (2005). Disponible en: http://i.unu.edu/media/unu.edu/publication/28551/antarctic_bioprospecting-3. pdf

Scott, Karen N., Managing Sovereignty and Jurisdictional Disputes in the Antarctic, Conferencia del 20 Aniversario de la Unidad de Investigación de Fronteras Internacionales, celebrada en Durham, Reino Unido entre el 1 y el 3 de abril de 2009. Disponible en: https://www.dur.ac.uk/resources/ibru/conferences/sos/ karen_scott_paper.pdf

\section{Working papers}

CoOlsaet, BRendan, Vers un partage équitable des avantages de la richesse génétique mondiale? Pouvoir et influence des pays les moins avancés dans la gouvernance internationale de la biodiversité (Dynamiques Internationales, Décembre, Working paper \#4, 2011). Disponible en: http://biogov.uclouvain.be/staff/ coolsaet $/ 2011 \% 20$ Coolsaet $\% 20-\% 20$ Vers $\% 20$ un $\% 20$ partage $\% 20$ equitable.pdf

\section{Tesis doctoral}

Davis, Jason Michael, Reconsidering Antarctic Bioprospecting through Territorialities of Science, Property and Governance (Tesis Doctoral, Ohio University, 2011). Disponible en: https://etd.ohiolink.edu/ap/10?0::NO:10:P10_ACCESSION_ NUM:osu1299535648

\section{Tratados, acuerdos y resoluciones internacionales}

Bonn Guidelines on Access to Genetic Resources and Fair and Equitable Sharing of the Benefits Arising Out of Their Utilization, 2002. Disponible en: https://www. cbd.int/abs/bonn/

Convención para la Conservación de las Focas Antárticas, Londres, 1972. Disponible en: http://www.ats.aq/documents/recatt/att076_s.pdf

Convención para la Conservación de los Recursos Vivos Marinos Antárticos, Camberra, 1980. Disponible en: http://www.ats.aq/documents/ats/ccamlr_s.pdf

Convenio sobre la Diversidad Biológica, Nairobi, 1992. Disponible en: https://www. cbd.int/convention/text/, https://www.cbd.int/doc/legal/cbd-es.pdf

Medidas Convenidas para la Protección de Flora y Fauna Antártica, Bélgica, 1964. Disponible en: http://www.ats.aq/documents/recatt\%5Catt080_s.pdf

Protocolo al Tratado Antártico sobre la Protección del Medio Ambiente - Protocolo de Madrid, Madrid, 1991. Disponible en: http://www.ats.aq/documents/recatt/ att006_s.pdf 
Tratado Antártico, suscrito en Washington, el 1 de diciembre de 1959. Disponible en: http://www.ats.aq/documents/ats/treaty_original.pdf

\section{Resoluciones del Sistema del Tratado Antártico}

Sistema del Tratado Antártico, Resolución 6, Prospección biológica en la Antártida (2013). Disponible en: http://ats.aq/documents/ATCM36/fr/ATCM36_fr001_s.pdf

Sistema del Tratado Antártico, Resolución 7, La prospección biológica en la Antártida (2005). Disponible en: http://www.ats.aq/documents/other/atcm28_res7_s.pdf

Sistema del Tratado Antártico, Resolución 9, Recolección y uso de material biológico antártico (2009). Disponible en: http://www.ats.aq/devAS/info_measures_ listitem.aspx?lang=s\&id=450

\section{Reuniones Consultivas del Tratado Antártico}

Reunión Consultiva del Tratado Antártico XXV (Varsovia, 2002), Bioprospecting in Antarctica, Working paper WP-043, Agenda Item CPA 4(d). Disponible en: http:// www.ats.aq/devAS/ats_meetings_documents.aspx?lang=s

Reunión Consultiva del Tratado Antártico XXV (Varsovia, 2002), Informe final. Disponible en: http://www.ats.aq/devAS/ats_meetings_meeting.aspx?lang=s

Reunión Consultiva del Tratado Antártico XXVI (Madrid, 2003), The International Regime for Bioprospecting: Existing Policies and Emerging Issues for Antarctica, Information Paper 075 UK/NO, Agenda Item VI CEP 7. Disponible en: http:// www.ats.aq/devAS/ats_meetings_documents.aspx?lang=e

Reunión Consultiva del Tratado Antártico XXVI (Madrid, 2003), Informe final. Disponible en: http://www.ats.aq/devAS/ats_meetings_meeting.aspx?lang=e

Reunión Consultiva del Tratado Antártico XXVII (Cape Town, 2004), Industry Involvement in Antarctic Bioprospecting. Information Paper 106 UNEP, Agenda Item CEP 7 and ATCM 17. Disponible en: http://www.ats.aq/devAS/ ats_meetings_documents.aspx?lang=e, http://www.unep.org/dewa/Portals/67/ pdf/bioprospecting_final.pdf

Reunión Consultiva del Tratado Antártico XXVII (Cape Town, 2004), Informe final. Disponible en: http://www.ats.aq/devAS/ats_meetings_meeting.aspx?lang=e

Reunión Consultiva del Tratado Antártico XXVIII (Stockholm, 2005), Information Paper 8, La bioprospección en la Antártida, Agenda Item ATCM18. Disponible en: http://www.ats.aq/devAS/ats_meetings_documents.aspx?lang=e

Reunión Consultiva del Tratado Antártico XXVIII (Stockholm, 2005), Informe final. Disponible en: http://www.ats.aq/devAS/ats_meetings_meeting.aspx?lang=e

Reunión Consultiva del Tratado Antártico XXVIII (Stockholm, 2005), La prospección biológica en la Antártida. Working Paper 13, Agenda Item ATCM18. Disponible en: http://www.ats.aq/devAS/ats_meetings_documents.aspx?lang=e 
Reunión Consultiva del Tratado Antártico XXIX (Edinburgh, 2006), Argentine Activities of Bioprospecting and Bioremediation in Antarctica, Information Paper 112, Agenda Item ATCM 18. Disponible en: http://www.ats.aq/devAS/ ats_meetings_documents.aspx?lang=e

Reunión Consultiva del Tratado Antártico XXIX (Edinburgh, 2006), Informe de un taller sobre especies no autóctonas en la Antártida, Working Paper 13, Agenda Item ATCM 8. Disponible en: http://www.ats.aq/devAS/ats_meetings_documents. aspx?lang $=\mathrm{s}$

Reunión Consultiva del Tratado Antártico XXIX (Edinburgh, 2006), Informe final. Disponible en: http://www.ats.aq/devAS/ats_meetings_meeting.aspx?lang=e

Reunión Consultiva del Tratado Antártico XXIX (Edinburgh, 2006), Progress During 2005-2006 in Implementing the International Polar Year 2007-2008, Information Paper 12, Agenda Item ATCM 18. Disponible en: http://www.ats.aq/devAS/ ats_meetings_documents.aspx?lang=s

Reunión Consultiva del Tratado Antártico XXIX (Edinburgh, 2006), Recent Trends in Biological Prospecting, Information Paper 116, Agenda Item ATCM 18. Disponible en: http://www.ats.aq/devAS/ats_meetings_documents.aspx?lang=e

Reunión Consultiva del Tratado Antártico XXX (New Delhi, 2007), Informe final. Disponible en: http://www.ats.aq/devAS/ats_meetings_meeting.aspx?lang=s

Reunión Consultiva del Tratado Antártico XXX (New Delhi, 2007), Revisión, actualización y mecanismo propuesto para apoyar la acción futura en materia de prospección biológica en la Antártida, Information Paper 67, Agenda Item 17. Disponible en: http://www.ats.aq/devAS/ats_meetings_documents.aspx?lang=s

Reunión Consultiva del Tratado Antártico XXXI (Kiev, 2008), Actualización sobre la bioprospección en la Antártida, incluida la creación de la Base de Datos sobre la Prospección Biológica en la Antártida, Working Paper 11, Agenda Item 17. Disponible en: http://www.ats.aq/devAS/ats_meetings_documents.aspx?lang=s

Reunión Consultiva del Tratado Antártico XXXI (Kiev, 2008), Informe final. Disponible en: http://www.ats.aq/devAS/ats_meetings_meeting.aspx?lang=s

Reunión Consultiva del Tratado Antártico XXXII (Baltimore, 2009), Actualización de las actividades del Programa Antártico Argentino sobre Bioprospección y Biorremediación en la Antártida, Information Paper 84, Agenda Item 17. Disponible en: http://www.ats.aq/devAS/ats_meetings_documents.aspx?lang=e

Reunión Consultiva del Tratado Antártico XXXII (Baltimore, 2009), Bioprospecting Activities of Brazil in Antarctica: A Short Report, Information Paper 115, Agenda Item 17. Disponible en: http://www.ats.aq/devAS/ats_meetings_documents. aspx?lang=e

Reunión Consultiva del Tratado Antártico XXXII (Baltimore, 2009), Update of the Activities of the Argentine Antarctic Program on Bioprospection and Bioremediation in the Antarctica, Information Paper 84, Agenda Item 17. Disponible en: http:// www.ats.aq/devAS/ats_meetings_documents.aspx?lang=e

Reunión Consultiva del Tratado Antártico XXXII (Baltimore, 2009), Informe final. Disponible en: http://www.ats.aq/devAS/ats_meetings_meeting.aspx?lang=e 
Reunión Consultiva del Tratado Antártico XXXIII (Punta del Este 2010), Informe de proyectos de bioprospección impulsados por el Ecuador, 2009-2010, Information Paper 125, Agenda Item 17. Disponible en: http://www.ats.aq/devAS/ats meetings_documents.aspx?lang=e

Reunión Consultiva del Tratado Antártico XXXIII (Punta del Este 2010), Informe final. Disponible en: http://www.ats.aq/devAS/ats_meetings_meeting.aspx?lang=e

Reunión Consultiva del Tratado Antártico XXXIII (Punta del Este, 2010), The Role of Ex-Situ Collections in Antarctic Bioprospecting, Information Paper 96, Agenda Item ATCM 17. Disponible en. http://www.ats.aq/devAS/ats_meetings_ documents.aspx?lang=e

Reunión Consultiva del Tratado Antártico XXXIV (Buenos Aires, 2011), Informe final. Disponible en: http://www.ats.aq/devAS/ats_meetings_meeting.aspx?lang=e

Reunión Consultiva del Tratado Antártico XXXIV (Buenos Aires, 2011), Informe sobre las recientes actividades de bioprospección desarrolladas por Argentina durante el período 2010-2011, Information Paper 16, Agenda Item 17. Disponible en: http:// www.ats.aq/devAS/ats_meetings_documents.aspx?lang=e

Reunión Consultiva del Tratado Antártico XXXV (Hobart, 2012), Management Plan for Romanian Biological Prospecting Activities in Antarctica, Information Paper 84, Agenda Item 18. Disponible en: http://www.ats.aq/devAS/ats_meetings_ documents.aspx?lang $=\mathrm{e}$

Reunión Consultiva del Tratado Antártico XXXV (Hobart, 2012), Informe final. Disponible en: http://www.ats.aq/devAS/ats_meetings_meeting.aspx?lang=e

Reunión Consultiva del Tratado Antártico XXXV (Hobart, 2012), Report on the Bioprospecting Activities Carried Out by Belgian Scientists since 1998, Information Paper 22, Agenda Item 18. Disponible en: http://www.ats.aq/devAS/ats_meetings_ documents.aspx?lang=e

Reunión Consultiva del Tratado Antártico XXXVI (Brussels, 2013), An Update on Status and Trends Biological Prospecting in Antarctica and Recent Policy Developments at the International Level. Information Paper 22, Agenda Item 17. Disponible en: http://www.ats.aq/devAS/ats_meetings_documents.aspx?lang=s

Reunión Consultiva del Tratado Antártico XXXVI (Brussels, 2013), www.biodiversity. aq La nueva red de información sobre biodiversidad antártica. Working Paper 37, Agenda Item 11. Disponible en: http://www.ats.aq/devAS/ats_meetings_ documents.aspx?lang=s

Reunión Consultiva del Tratado Antártico XXXVI (Brussels, 2013), Biological Prospecting in Antarctica - the Need for Improved Information. Working Paper 48, Agenda Item 17. Disponible en: http://www.ats.aq/devAS/ats_meetings_ documents.aspx?lang=e

Reunión Consultiva del Tratado Antártico XXXVI (Brussels, 2013), Informe final. Disponible en: http://www.ats.aq/devAS/ats_meetings_meeting.aspx?lang=e 
